\title{
An ontology-driven communication architecture for spontaneous interoperability in Home Automation systems
}

\author{
J. Juárez, J. A. Rodríguez-Mondéjar \\ Comillas Pontifical University, Institute for Research in \\ Technology (IIT), 28015 Madrid, Spain \\ javier.juarez@iit.upcomillas.es \\ mondejar@dea.icai.upcomillas.es
}

\author{
R. García-Castro \\ Ontology Engineering Group, Escuela Técnica Superior de \\ Ingenieros Informáticos, Universidad Politécnica de Madrid, \\ Madrid, Spain \\ rgarcia@fi.upm.es
}

\begin{abstract}
Current solutions to the interoperability problem in Home Automation systems are based on a priori agreements where protocols are standardized and later integrated through specific gateways. In this regards, spontaneous interoperability, or the ability to integrate new devices into the system with minimum planning in advance, is still considered a major challenge that requires new models of connectivity. In this paper we present an ontology-driven communication architecture whose main contribution is that it facilitates spontaneous interoperability at system model level by means of semantic integration. The architecture has been validated through a prototype and the main challenges for achieving complete spontaneous interoperability are also evaluated.
\end{abstract}

\section{INTRODUCTION}

The Internet of Things (IoT), which aims to connect physical devices over the Internet, is starting to cause a high impact in everyday-life of people. Particularly, traditional Home Automation systems are evolving towards more intelligent systems where a huge amount of functionalities are available to users. The great heterogeneity of devices from different manufacturers (i.e. home appliances, sensors, actuators, mobile phones, wearable devices, etc.), technologies (i.e. wireless, power-line communication, etc.), and protocols (i.e. ZigBee SEP, ZigBee HA, Z-Wave, etc.) coexisting together, has led interoperability to be one of the main challenges to be overcome by IoT systems in order to reach its full potential.

Current solutions to this problem are oriented towards a priori agreements where protocols and models are standardized and later integrated through specific gateways. Every time a new unplanned device, technology or protocol is going to join to a system, it must be explicitly incorporated into some gateway, being difficult to automate the process. In this regard, spontaneous interoperability, or the ability to integrate new devices into the system with minimum planning in advance, is considered a major challenge and requires new models of connectivity that reach beyond simple prior agreements on standard protocols [1].
It is worth noting that there are a growing number of systems employing ontologies (ontology-driven systems) as semantic descriptions for modeling their characteristics and functionalities independently from technology-specific issues. This type of systems bases their connectivity model in messages that contain expressions formulated in terms of their ontologies (ontology-driven communication).

The use of ontologies provides a lot of advantages to Home Automation systems (e.g. they allow to reason about the available information and to conduct complex queries) but, in order to achieve spontaneous interoperability, all the device manufacturers and system developers should share the same ontology (both at design time and at run time). Taking into account the large number of ontologies that have emerged recently, this possibility can be considered unrealistic. Therefore, ontologies do not actually solve the device and technology heterogeneity problem, they just move it to a higher level.

In this paper we present an ontology-driven communication architecture whose main contribution is that it facilitates spontaneous interoperability at system model level by means of semantic integration (i.e. devices can exchange data without the need to standardize their information models). This architecture is validated through a prototype and the main challenges for achieving complete spontaneous interoperability are also evaluated.

The rest of the paper is structured as follows. Section II introduces other ontology-driven information systems. Section III presents works related to the use of ontologies in Home Automation systems. Section IV describes the proposed architecture. Section V validates it through a prototype. Finally, Section VI concludes the paper.

\section{ONTOLOGY-DRIVEN SYSTEMS}

One of the most extended definitions of ontology was proposed by Gruber [2], and completed by Borst [3]: An ontology is a formal, explicit specification of a shared conceptualization. For Gruber, a conceptualization is an abstract, simplified view of the world that we wish to represent for any purpose. Other definitions of ontology include their relationship with Knowledge Bases (KBs). A KB 
is a collection of knowledge expressed using some formal representation language and constructed in a way that an application can use it to carry out inferences. According to Swartout et al. [4], an ontology is a hierarchically structured set of terms for describing a domain that can be used as a skeletal foundation for a knowledge base. Thus, an ontology together with a set of instances constitutes a KB [5].

Ontology-driven information systems, as presented by Guarino [6], are those that use ontologies for managing their knowledge, enabling the communication between agents at run time. In order to achieve a successful ontology-driven communication, each agent should be able to understand the information provided by others. If they use the same ontology, or at least ontologies based on the same vocabulary, they will be able to comprehend that information unequivocally. On the other hand, if they do not share the same ontology, in order to solve the semantic heterogeneity problem, they need to adopt some mechanism that aligns the concepts of the ontologies, and allows agents to understand each other.

\section{RELATED WORK}

There are a lot of systems that follow architectures based on ontologies for the semantic representation of their devices and services. For instance, Bonino et al. [7] present a gateway based on OSGi middleware, where heterogeneous devices and systems are integrated by means of an ontology. They model the home structure, its components, their states and functionalities by defining a custom ontology called DogOnt [8].

In general, these approaches use gateways where device data have to be transformed to a semantic format in order to achieve the interoperability. As pointed out by Kiljander et al. [9] these solutions make the embedded system side easy to implement (since they do not need to support semantic technologies) but, on the other hand, gateways have to be modified each time an unplanned device, technology or protocol is going to be integrated on the system leading to scalability problems. The same authors propose an approach based on sharing semantic data via knowledge bases integration. They have developed their approach on top of the Smart-M3 semantic interoperability platform [10]. The solutions based on this platform have the advantage that, since all devices utilize the same ontology, it is possible for them to exchange and interpret their data mutually.

The aforementioned approaches are based on the use of a common ontology for providing a unified view of the system, its services and its devices. However, in Home Automation systems where different parties are usually involved, it is expected that different manufacturers employ different ontologies without previous agreement, conducting to a semantic heterogeneity problem that has to be overcome in order to facilitate spontaneous interoperability. The architecture presented here differs from the above solutions in that the semantic description of systems does not need previous ontology standardization in order to interoperate.

\section{ARCHITECTURE}

Although the main objective of the architecture we present is to facilitate spontaneous interoperability, it is just achieved at system model level, and therefore it is still necessary to establish a minimum consensus about the technologies and standards employed. The language used for representing ontologies is OWL, and the communication interface is based in the HTTP methods and JSON messages. The reason to use JSON is that it is a lightweight, text-based, languageindependent data interchange format [11], which has been designed in order to be more human readable and ease for computers to parse than other XML languages [12].

The architecture is based on a central gateway called SeSGat (Semantic Smart Gateway), whose main role is to interconnect systems and devices and to integrate spontaneously the information they provide, such as states or consumptions, in a unified way.

The core of SeSGat is an ontology called SHOnt ${ }^{1}$ (Smart Home Ontology) whose main objectives are to provide background knowledge to the ontology matching process, and to offer a unified view of Smart Home systems. It has been developed analyzing the most representative ontologies from areas such as Home Automation, context-aware pervasive systems, ambient intelligence systems, activity recognition systems and Smart Energy systems.

The architecture of SeSGat is shown in Fig.1.

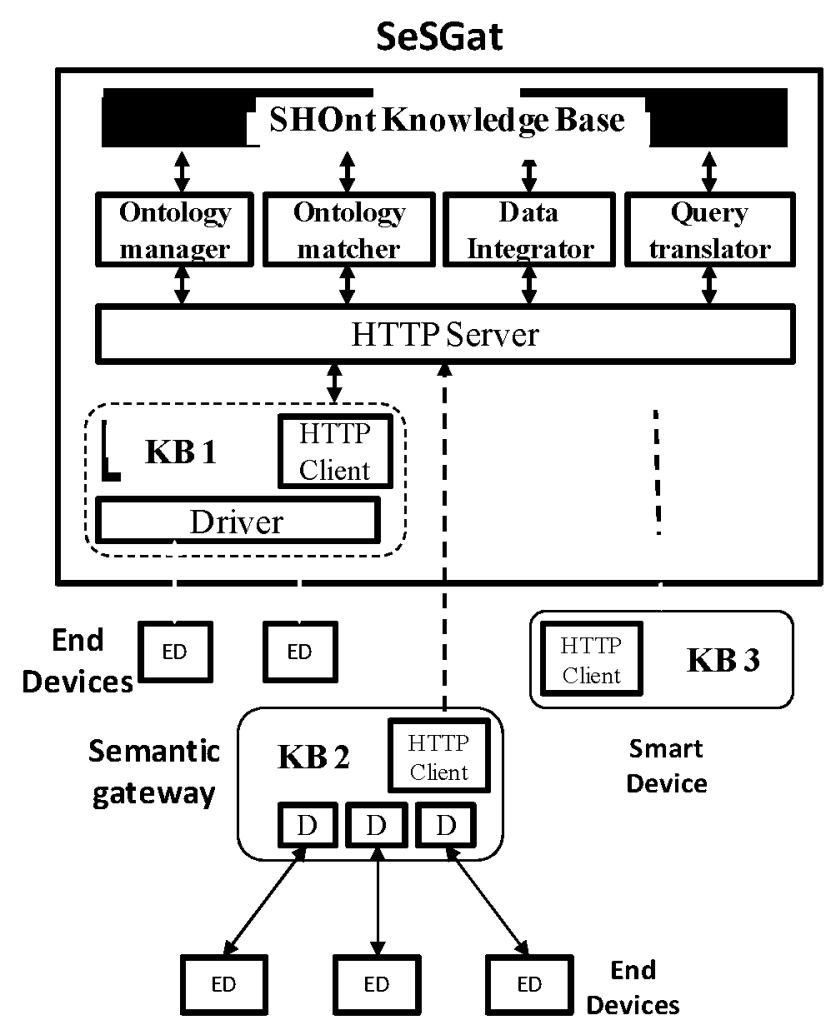

Fig. 1. SeSGat architecture. 
It is also composed by an ontology manager for handling SHOnt, an ontology matcher for performing the ontology matching process when a new system or device is subscribed to SeSGat, a data integrator that translates and integrates new data into the SHOnt $\mathrm{KB}$, and a query translator, which translate queries based on a specific ontology in queries expressed in terms of SHOnt using the Manchester OWL syntax [13], a quick and easy to read and write syntax.

SeSGat contemplates the integration of low-capacity devices, other independent semantic gateways, and smart devices that contain their own semantic descriptions.

Each time a new system wants to subscribe to SeSGat it has to share its own KB, that is to say, the ontology, as reference model, and the instances that represents its data. Then, the ontology matcher tries to find the correspondences between the ontology and SHOnt generating an alignment file. This process is considered one of the most relevant parts of the integration. The matcher employs a set of algorithms for exploiting efficiently the background knowledge provided by SHOnt, not just in order to discover new correspondences missed by other approaches, but to detect and to correct false alignments. Once the ontologies are aligned, the data integrator transforms instances from the ontology into corresponding instances expressed in terms of SHOnt.

All agents in SeSGat can update their data, or access the information contained in SHOnt by means of description logic queries expressed using its own terms.

\section{Evaluation OF THE ARCHITECTURE}

SeSGat has been implemented using the Raspberry $\mathrm{Pi}^{2}$ model B, a low cost, credit-card sized computer. Fig. 2 shows the evaluated scenario. It is composed by the Raspberry Pi, a mobile phone with Android operating system, a LDR sensor for measuring luminance, a DHT11 sensor for measuring temperature and humidity, an Arduino ${ }^{3}$ UNO for controlling the sensors, a ZigBee ${ }^{4}$ coordinator form Radiocrafts ${ }^{5}$ with Smart Energy (SE) profile, and a ZigBee SE Smart Plug form SimpleHomeNet ${ }^{6}$.

The driver for the ZigBee coordinator is installed inside SeSGat and uses BOnSAI [14], an ontology developed by the Aristotle University of Thessaloniki, in order to represent the ZigBee network information. The ZigBee SE device is connected to a simple lamp for monitoring its consumption. The driver for controlling the sensors through the Arduino, uses DogOnt [8], an ontology developed by Politecnico di Torino.

The information provided by sensors and devices, even if they use different ontology-based models for representing their data, is automatically integrated into SeSGat by means of translating data to the SHOnt ontology. All the system and devices subscribed to SeSGat can access this information.

\footnotetext{
2 http://www.raspberrypi.org/

3 http://www.arduino.cc/

4 https://www.zigbee.org/

5 http://www.radiocrafts.com/

${ }^{6}$ https://simplehomenet.com/
}

Regarding to this, we have also developed an Android application based on SHOnt, which monitors the states, consumptions and parameters of the sensor and devices connected to SeSGat.

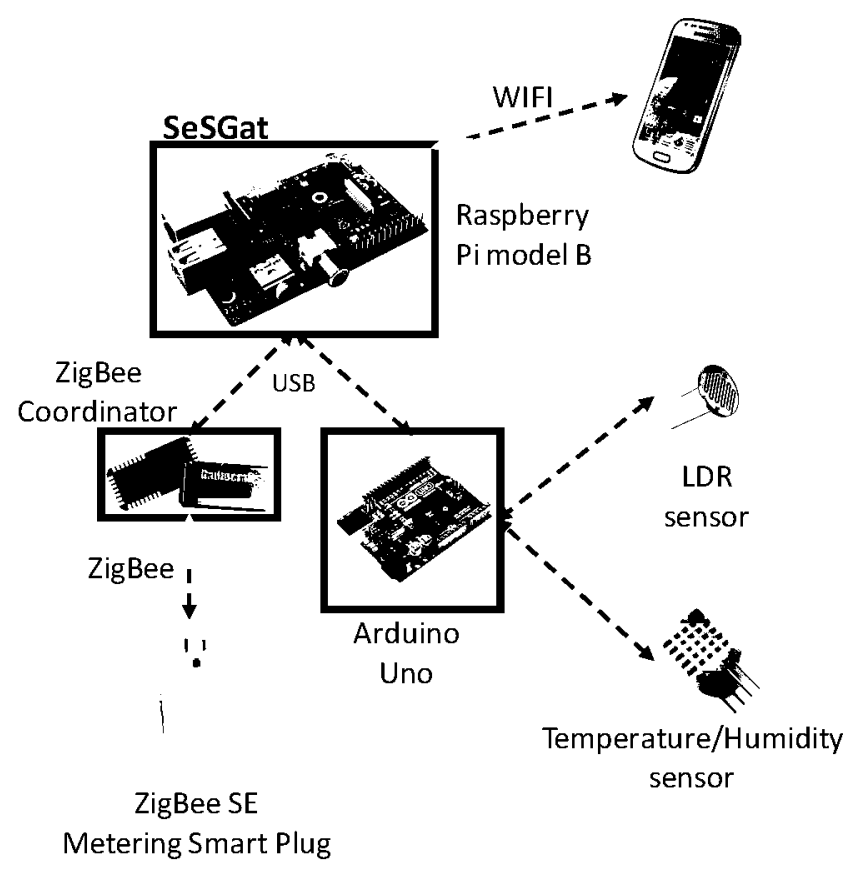

Fig. 2. Evaluated scenario.

\section{A. Challenges}

The evaluation of the proposed architecture has shown a set of challenges necessary to solve in order to achieve the complete spontaneous interoperability at model level.

\section{1) Improvements of the ontology matching process}

Although the ontology matching system developed here shows good results regarding completeness and precision, it has not yet achieved to integrate the $100 \%$ of the information, and there has been some loss of information in the data translation due to undetected correspondences between ontologies. Besides, some false correspondences have also been detected. These mismatches have been manually corrected, but in order to avoid unwanted behaviors of the systems, it is necessary to develop some mechanism that corrects and minimizes these errors automatically.

\section{2) Harmonization of different conceptualizations}

Ontologies with different conceptualizations usually represent the same concepts in ways that make not possible to integrate them directly. For instance, some ontologies can represent the state of a device with a simple data attribute of a Device class, while others use a different class for representing states and then relate it with Device through a specific object property.

Aiming to achieve spontaneous interoperability, it is necessary to develop some mechanism that carries out this harmonization automatically. 


\section{3) Correction}

of

incompatibilities

between conceptualizations

Some incompatibilities between ontologies regarding their conceptualizations have been detected during the evaluation of the architecture. For instance, according to BOnSAI, an actuator can also be a sensor but for DogOnt this is not possible. This can create an inconsistency in the DogOnt side when it tries to access sensors and actuators of the BOnSAI side. These problems need to be avoided.

\section{CONCLUSIONS}

This paper introduces SeSGat, an ontology-driven communication architecture, whose main objective is to facilitate spontaneous interoperability at system model level by means of semantic integration. This architecture has been validated through a prototype developed with microcontrollerbased platforms such as Raspberry $\mathrm{Pi}$ and Arduino. The sensors and devices integrated were based in two different ontology-based models.

We have also identified and evaluated three challenges necessary to solve in order to achieve complete spontaneous interoperability at system model level.

\section{References}

[1] W. Edwards and R. Grinter, "At home with ubiquitous computing: Seven challenges", in Ubicomp 2001: Ubiquitous Computing, 2001, pp. 256-272.

[2] T. R. Gruber "A translation approach to portable ontology specifications", Knowledge acquisition, vol. 5, no. 2, pp. 199220,1993

[3] W. N. Borst, "Construction of Engineering Ontologies for Knowledge Sharing and Reuse", PhD Thesis, University of Twente, Enschede, 1997.
[4] B. Swartout, R. Patil, K. Knight, and T. Russ, "Toward distributed use of large-scale ontologies", in Proc. of the Tenth Workshop on Knowledge Acquisition for Knowledge-Based Systems, 1996.

[5] N. F. Noy and D. L. McGuinness, Ontology development 101: A guide to creating your first ontology. Stanford knowledge systems laboratory technical report KSL-01-05 and Stanford medical informatics technical report SMI-2001-0880, 2001.

[6] N. Guarino, "Formal Ontology in Information Systems", in Proceedings of the first international conference on formal ontology in information systems, Trento, Italy, 1998, pp. 3-15.

[7] D. Bonino, E. Castellina, and F. Corno, "The DOG gateway: enabling ontology-based intelligent domotic environments", IEEE Transactions on Consumer Electronics, vol. 54, no. 4, pp. 1656-1664, Nov. 2008

[8] D. Bonino and F. Corno, "Dogont-ontology modeling for intelligent domotic environments", The Semantic Web-ISWC, pp. $790-803,2008$.

[9] J. Kiljander et al., "Enabling Semantic Technology Empowered Smart Spaces", Journal of Computer Networks and Communications, vol. 2012, Dec. 2012.

[10] J. Honkola, H. Laine, R. Brown, and O. Tyrkko, "Smart-M3 Information Sharing Platform", in Proceedings of the The IEEE Symposium on Computers and Communications, Washington, DC, USA, 2010, pp. 1041-1046.

[11] D. Crockford, "The application/json Media Type for JavaScript Object Notation (JSON)". RFC 4627, Jul-2006.

[12] N. Nurseitov, M. Paulson, R. Reynolds, and C. Izurieta, "Comparison of JSON and XML Data Interchange Formats: A Case Study", in CAINE, 2009, pp. 157-162.

[13] M. Horridge, N. Drummond, J. Goodwin, A. Rector, and H. H. Wang, "The Manchester OWL syntax", in Proceedings of the OWL Experiences and Directions Workshop (OWLED'06) at the ISWC'06, 2006

[14] T. G. Stavropoulos, D. Vrakas, D. Vlachava, and N. Bassiliades, "BOnSAI: a smart building ontology for ambient intelligence", in Proceedings of the 2nd International Conference on $\mathrm{We} b$ Intelligence, Mining and Semantics, 2012, p. 30. 